
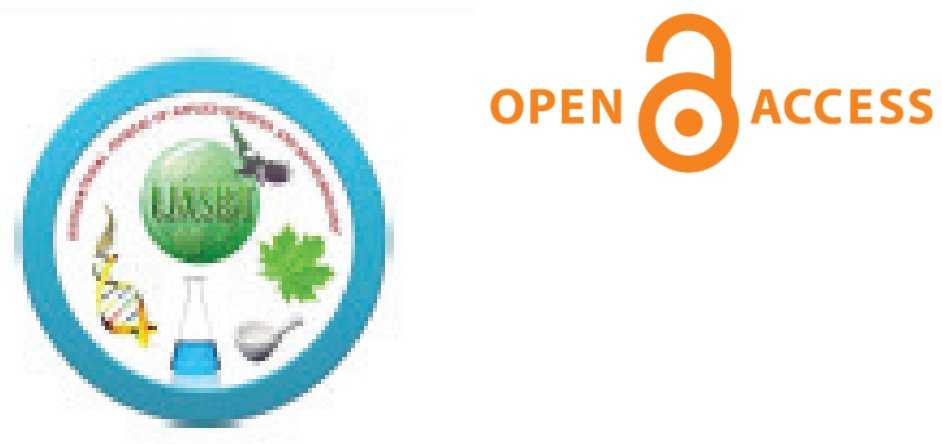

Avalable online at wwwijastht.org

International Journal of Applied Sciences and Biotechnology A Rapid Publishing Journal

\begin{tabular}{|c|c|c|}
\hline \multicolumn{2}{|c|}{ APPUED SCIENCES } & DIOTECHNOLOGY \\
\hline $\begin{array}{l}\text { Blochemistry } \\
\text { Molecular blology } \\
\text { Microbiolow } \\
\text { Cell blalogy }\end{array}$ & $\begin{array}{l}\text { Immunobiology } \\
\text { Biolnformatics } \\
\text { Nowel drug delivery rytem } \\
\text { Pharmacology }\end{array}$ & $\begin{array}{l}\text { Mierob al biotechnolosy } \\
\text { Med lal biotechnology } \\
\text { Industrial blotechnolosy } \\
\text { Enviranmental bilotechnolosy }\end{array}$ \\
\hline $\begin{array}{l}\text { Cytology } \\
\text { Genetic } \\
\text { Patholosy } \\
\text { Medicinal chemistry } \\
\text { Polymer sdences } \\
\text { Analytical chemistry } \\
\text { Natural chemistry }\end{array}$ & $\begin{array}{l}\text { Neuroblalogy } \\
\text { Bio-physlcs } \\
\text { Botany } \\
\text { Zoology } \\
\text { Allied science } \\
\text { Earth sdence }\end{array}$ & Nanotechnology \\
\hline
\end{tabular}

If any queries or feedback, then don't hesitate to mail us at: editor.ijasbt@email.com 


\title{
STUDY ON THERMOPHILES REVEALS THE PRESENCE OF ACTINOBACILLUS LIGNIERESII IN CATTLE COMPOST
}

\author{
Gagandeep Singh Saggu" and Divya Shrivastava ${ }^{2}$ \\ ${ }^{1}$ Department of Biological Sciences, Birla Institute of Technology and Science, Pilani, Rajasthan, India \\ ${ }^{2}$ School of Life Sciences, Jaipur National University, Jaipur, Rajasthan, India \\ Corresponding Author email: gagandeep.saggu@pilani.bits-pilani.ac.in
}

\begin{abstract}
Introduction- Moderate environment is important to sustain life any environment condition that can be perceived as beyond the normal acceptable range is extreme condition and if we are talking in the terms of temperature than its called thermophillic environment. Aim \& objective- The study was designed to study the diversity of thermophiles present in compost and characterization of the properties which are responsible for their adequate nature to sustain high temperature. Material and methodMicroorganisms were isolated from cattle compost and initially screened by biochemical tests to characterize till genus level. Confirmation of Actinobacillus spp. was done by fermentation reactions of nine carbohydrates and amplification of 16S rRNA region. Results- Isolates from compost showed wide range of growth temperatures which suggest their important activity during the whole composting process. This study initially designed to study the diversity of thermophiles in compost which turned specifically to A. lignieresii.
\end{abstract}

Key words- compost, thermophiles, Actinobacillus, biochemical, molecular analysis.

\section{Introduction}

Moderate environment is important to sustain life. Moderate means environments with near neutral $\mathrm{pH}$, temperature between 20 and $40^{\circ} \mathrm{C}$, air pressure $1 \mathrm{~atm}$ and adequate levels of available water, nutrients and salts. Any environmental condition that can be perceived as beyond the normal acceptable range is an extreme condition. However, a variety of microorganisms survive in those conditions also. These organisms not only tolerate but require such conditions for their growth and survival. Such organisms are called extremophiles (Satyanarayana, 2005). Of these extremophiles, organisms which survive in high temperature areas are called thermophiles. This type of organisms is further classified as Obligate, which require high temperature for their survival, and moderate, which can thrive at high temperatures but also at lower temperature. For an organism to grow at high temperatures, especially as high as those of the hyperthermophiles discussed here, all cellular components, including proteins, nucleic acids, and lipids, must be heat stable (Brock,1967; Brock et al., 1970). The thermal stabilities of enzymes from various hyperthermophiles are referred as extremozymes (Gomes et al., 2004) and some of such enzymes have been found to remain active up to $140^{\circ} \mathrm{C}$ (Ladenstein et al., 1998). Besides temperature, other environmental parameters such as $\mathrm{pH}$, available energy sources, ionic strength and nutrients also influence the population of thermophiles. The structural features that dictate thermal stability in proteins are not well understood but a small number of noncovalent features seem characteristic of thermostable proteins. These include a highly apolar core, which undoubtedly makes the inside of the protein "sticky" and thus more resistant to unfolding, a small surface-to-volume ratio, which confers a compact form on the protein, a reduction in glycine content that tends to remove options for flexibility and thus introduce rigidity to the molecule, and extensive ionic bonding across the protein's surface that helps the compacted protein resist unfolding at high temperature. In addition to these intrinsic stability factors, special proteins called chaperonins are synthesized by hyperthermophiles, which functions to bind with heat denatured proteins and refolds them into their active form. Thermophiles can ferment similar carbohydrates, utilize similar nitrogen sources, and have similar oxidative pathways. They can exist as aerobes, anaerobes, or as facultative aerobes. There are also autotrophic and heterotrophic species in 
thermophiles. The search for extremophilic organism is one of the means for obtaining enzymes with properties suitable for industrial purposes (Ibrahim et al., 2007; Turner et al., 2007). Such enzymes have found their way into the grist of industry in applications as diverse as laundry detergent additives (proteases, lipases) and the genetic identification of criminals. DNA polymerases have been obtained from Thermococcus littoralis, Thermus aquaticus, Thermotoga maritime, Pyrococcus woesii and P. furiosus for application in polymerase chain reaction (PCR). Another important realization that has emerged from the study of extremophiles is that some of these organisms form the cradle of life itself. Many extremophiles, in particular the hyperthermophiles, lie close to the "universal ancestor" of all extant life on Earth. Thus, an understanding of the basic biology of these organisms is an opportunity for biologists to "look backward in time" so to speak, to a period of early life on Earth. Thermophiles are found in various geothermally heated regions of the earth such as hot springs like those in Yellowstone National Park and deep sea hydrothermal vents, as well as decaying plant matter such as peat bogs and compost.

Thermophiles presence can also be seen in compost samples because during composting temperature increase to level where only thermophiles can survive. Composting is the aerobic decomposition of organic materials by microorganisms under controlled conditions into a soil-like substance called compost. During composting, microorganisms such as bacteria and fungi break down complex organic compounds into simpler substances and produce carbon dioxide, water, minerals, and stabilized organic matter (Suler et al., 1977). The composting process at the microbial level involves several interrelated factors, i.e metabolic heat generation, temperature, ventilation, moisture content, and available of nutrients. The temperature both reflects prior microbial activity and current rate of activity. The initial rapid increase of temperature involves a rapid transition from a mesophilic to a thermophilic microflora (Strom, 1985a; Strom, 1985b). The compost ecosystem then tends to limit itself due to inhibitory high temperatures, resulting from excessive heat accumulation (Nakasaki et al., 1985a; Nakasaki et al., 1985b). Aiming isolation of thermophilic microorganism from compost ecosystem will provide a good range of organisms which can survive at the compost temperature (Schulze, 1962).

\section{Materials and methods}

\section{Sample collection}

The cattle waste compost samples were collected from (Jaipur, Rajasthan), India. These samples were stored at $60^{\circ} \mathrm{C}$ and used for microbiological and analytical study of thermophilic bacteria.

\section{Screening and isolation of thermophilic bacteria}

The samples were suspended and diluted serially for 5 times in sterile distilled water. $100 \mu \mathrm{l}$ of each dilution were plated on a nutrient agar plate by using pour plate method and incubated at $60^{\circ} \mathrm{C}$ for $16-18 \mathrm{hr}$. Morphologically different colonies were picked up, checked for their purity and lyophilized in skim milk. After confirmation, each Actinobacillus isolates were maintained on blood agar plates.

\section{Characterization of colonies}

Individual colonies were characterized on the basis of colony morphology (shape, size, texture and colour), gram staining and conventional biochemical tests. Single colonies were obtained by using streak plate method. The isolated colony was streaked on nutrient agar slants and blood agar plates and incubated at $60^{\circ} \mathrm{C}$ for $16 \mathrm{hr}$ to obtain optimum growth. The preliminary characterization was based on colony morphology on blood agar plates after $16-18 \mathrm{hr}$ of incubation at $60^{\circ} \mathrm{C}$.

\section{Physiological characterization of the isolates}

Growth of the isolates was assessed in Nutrient broth at different temperature i.e. $35,55,60$ and $70^{\circ} \mathrm{C}$ and different $\mathrm{pH} 3,5,7,9$ and 11 by incubating at $60^{\circ} \mathrm{C}$. Salt tolerance was tested by incorporating 1, 2, 3, 5 and $7 \%(\mathrm{~W} / \mathrm{V})$ sodium chloride in NB.

\section{Biochemical Analysis of the bacterial isolates}

Individual colonies were characterized by morphological and conventional biochemical tests like Gram staining, Endospore staining, Motility test, Catalase activity, Starch hydrolysis, Citrate utilization test, Urease test, Oxidase test, Indole test, MRVP test, Nitrate reductase test and Triple sugar iron agar test. These tests were performed according to Microbiology laboratory manual $4^{\text {th }}$ edition by Cappuccino and Sherman (1999). Tentative identification of all bacterial colonies was done with the above tests (Table $1-5)$. 
Saggu GS and Shrivastava D (2013). Int J Appl Sci Biotechnol, Vol. 1(2): 33-41

Table 1: Biochemical analysis for tentative identification of isolated organism

\begin{tabular}{|c|c|c|c|c|c|c|c|c|c|}
\hline \multicolumn{2}{|l|}{ Isolate } & A1 & A3 & A4 & A5 & A6 & A7 & A8 & A9 \\
\hline \multicolumn{2}{|c|}{ Gram's staining } & $\begin{array}{l}\text { Gram } \\
\text { negative, } \\
\text { long rods, } \\
\text { bunches, } \\
\text { endospore } \\
\text { forming }\end{array}$ & $\begin{array}{l}\text { Gram } \\
\text { negative, } \\
\text { small rods, } \\
\text { bunches }\end{array}$ & $\begin{array}{l}\text { Gram } \\
\text { negative, } \\
\text { small rods, } \\
\text { bunches \& } \\
\text { chain }\end{array}$ & $\begin{array}{l}\text { Gram } \\
\text { positive, long } \\
\text { rods, } \\
\text { bunches, } \\
\text { endospore } \\
\text { forming }\end{array}$ & $\begin{array}{l}\text { Gram } \\
\text { negative, } \\
\text { rods, } \\
\text { bunches }\end{array}$ & $\begin{array}{l}\text { Gram } \\
\text { positive, } \\
\text { long rods, } \\
\text { single, } \\
\text { endospore } \\
\text { forming }\end{array}$ & $\begin{array}{l}\text { Gram } \\
\text { negative, } \\
\text { short } \\
\text { rods, } \\
\text { single \& } \\
\text { bunches, } \\
\text { endospore } \\
\text { forming }\end{array}$ & $\begin{array}{l}\text { Gram } \\
\text { negative, } \\
\text { rods, } \\
\text { single \& } \\
\text { chain } \\
\text { endospore } \\
\text { forming }\end{array}$ \\
\hline \multicolumn{2}{|c|}{ Endospore staining } & + & - & - & + & - & + & + & + \\
\hline \multicolumn{2}{|c|}{ Motility } & - & - & - & + & - & + & + & - \\
\hline \multicolumn{2}{|l|}{ Catalase } & + & - & - & - & + & + & + & + \\
\hline \multicolumn{2}{|c|}{ Starch hydrolysis } & - & - & - & + & + & + & - & + \\
\hline \multicolumn{2}{|c|}{ Citrate } & - & - & - & - & - & - & - & - \\
\hline \multicolumn{2}{|l|}{ Urease } & - & - & + & - & - & - & - & - \\
\hline \multicolumn{2}{|l|}{ Oxidase } & + & + & - & - & + & + & - & - \\
\hline \multicolumn{2}{|l|}{ Indole } & - & - & - & + & - & - & - & - \\
\hline \multicolumn{2}{|c|}{ Methyl red } & - & - & - & - & - & - & - & - \\
\hline \multicolumn{2}{|c|}{ Voges Proskauer } & - & - & - & - & - & - & - & - \\
\hline \multirow[t]{2}{*}{$\begin{array}{l}\text { Nitrate } \\
\text { reductase }\end{array}$} & $\begin{array}{l}\text { Without } \\
\mathrm{Zn}\end{array}$ & + & - & - & - & - & + & - & + \\
\hline & $\begin{array}{l}\text { With } \\
\text { Zn }\end{array}$ & & + & + & + & + & & + & \\
\hline \multirow{3}{*}{$\begin{array}{l}\text { Triple } \\
\text { sugar } \\
\text { iron agar }\end{array}$} & Glucose & - & + & + & - & - & & & - \\
\hline & Lactose & - & + & - & - & - & & & - \\
\hline & Sucrose & - & + & - & - & - & & & - \\
\hline \multicolumn{2}{|c|}{$\begin{array}{l}\text { Tentative } \\
\text { identification }\end{array}$} & Brahnamella & $\begin{array}{l}\text { Actinobacillus } \\
\text { lignieressi }\end{array}$ & $\begin{array}{l}\text { Pasteurella } \\
\text { ureae }\end{array}$ & $\begin{array}{l}\text { Streptococcus } \\
\text { cremoris }\end{array}$ & $\begin{array}{l}\text { Pasteurella } \\
\text { haemolytica }\end{array}$ & Micrococcus & $\begin{array}{l}\text { Erwinia } \\
\text { herbicola }\end{array}$ & Bordetella \\
\hline \multicolumn{2}{|c|}{ Similarity index } & 0.93193 & 0.28138 & 0.44953 & & 0.74153 & 0.58468 & 0.52585 & 0.786 \\
\hline
\end{tabular}

Table 2: Biochemical analyses for tentative identification of isolated organism

\begin{tabular}{|c|c|c|c|c|c|c|c|c|c|}
\hline \multicolumn{2}{|c|}{ Isolate } & A10 & A11 & A13 & A14 & A15 & A17 & A18 & A19 \\
\hline \multicolumn{2}{|c|}{ Gram's staining } & $\begin{array}{l}\text { Gram } \\
\text { negative, } \\
\text { short rods, } \\
\text { single \& } \\
\text { bunches }\end{array}$ & $\begin{array}{l}\text { Gram } \\
\text { positive, } \\
\text { long rods, } \\
\text { single \& } \\
\text { group }\end{array}$ & $\begin{array}{l}\text { Gram } \\
\text { positive, } \\
\text { long rods, } \\
\text { single \& } \\
\text { group }\end{array}$ & $\begin{array}{l}\text { Gram } \\
\text { negative, } \\
\text { small rods, } \\
\text { groups, } \\
\text { endospore } \\
\text { forming }\end{array}$ & $\begin{array}{l}\text { Gram } \\
\text { negative, } \\
\text { small rods, } \\
\text { bunch }\end{array}$ & $\begin{array}{l}\text { Gram } \\
\text { positive, } \\
\text { long rods, } \\
\text { endospore } \\
\text { forming }\end{array}$ & $\begin{array}{l}\text { Gram } \\
\text { negative, } \\
\text { small rods, } \\
\text { bunch }\end{array}$ & $\begin{array}{l}\text { Gram } \\
\text { positive, } \\
\text { single rods, } \\
\text { endospore } \\
\text { forming }\end{array}$ \\
\hline \multicolumn{2}{|c|}{ Endospore staining } & - & - & - & + & - & + & - & + \\
\hline \multicolumn{2}{|c|}{ Motility } & - & - & + & - & - & + & + & + \\
\hline \multicolumn{2}{|c|}{ Catalase } & - & + & - & + & + & + & - & - \\
\hline \multicolumn{2}{|c|}{ Starch hydrolysis } & - & + & + & + & - & - & - & - \\
\hline \multicolumn{2}{|c|}{ Citrate } & - & - & - & - & - & - & - & - \\
\hline \multicolumn{2}{|l|}{ Urease } & - & - & - & - & - & + & + & - \\
\hline \multicolumn{2}{|l|}{ Oxidase } & + & + & - & + & + & - & - & - \\
\hline \multicolumn{2}{|c|}{ Indole } & - & - & + & - & - & - & - & - \\
\hline \multicolumn{2}{|c|}{ Methyl red } & - & - & - & - & - & - & - & - \\
\hline \multicolumn{2}{|c|}{ Voges Proskauer } & - & - & - & - & - & - & - & - \\
\hline \multirow[t]{2}{*}{$\begin{array}{l}\text { Nitrate } \\
\text { reductase }\end{array}$} & $\begin{array}{l}\text { Without } \\
\mathrm{Zn}\end{array}$ & - & + & + & + & - & - & - & - \\
\hline & With Zn & + & & & & + & - & + & + \\
\hline \multirow{4}{*}{\multicolumn{2}{|c|}{$\begin{array}{ll}\text { Triple } & \text { Glucose } \\
\text { sugar } & \text { Lactose } \\
\text { iron agar } & \text { Sucrose } \\
\text { Tentative } & \text { identification }\end{array}$}} & + & - & - & - & - & - & + & - \\
\hline & & + & - & - & - & + & - & + & - \\
\hline & & + & - & - & - & + & - & + & - \\
\hline & & $\begin{array}{l}\text { Actinobacill } \\
\text { us } \\
\text { lignieressi }\end{array}$ & $\begin{array}{l}\text { Not } \\
\text { identified }\end{array}$ & $\begin{array}{l}\text { Not } \\
\text { identified }\end{array}$ & $\begin{array}{l}\text { Pasturella } \\
\text { haemolytica }\end{array}$ & $\begin{array}{l}\text { Pasturella } \\
\text { haemolytica }\end{array}$ & $\begin{array}{l}\text { Not } \\
\text { identified }\end{array}$ & $\begin{array}{l}\text { Actinobacillus } \\
\text { lignieressi }\end{array}$ & Micrococcus \\
\hline \multicolumn{2}{|c|}{ Similarity index } & 0.73694 & & & 0.40920 & 0.39572 & & 0.94647 & \\
\hline
\end{tabular}


Table 3: Biochemical analyses for tentative identification of isolated organism

\begin{tabular}{|c|c|c|c|c|c|c|c|c|c|}
\hline \multicolumn{2}{|l|}{ Isolate } & A20 & A21 & A22 & $\mathbf{A 2 3}$ & A25 & A26 & A28 & A29 \\
\hline \multicolumn{2}{|c|}{ Gram's staining } & $\begin{array}{l}\text { Gram } \\
\text { positive } \\
\text { bacilli, } \\
\text { single \& } \\
\text { bunches }\end{array}$ & $\begin{array}{l}\text { Gram } \\
\text { negative, small } \\
\text { rods, bunches }\end{array}$ & $\begin{array}{l}\text { Gram } \\
\text { negative, } \\
\text { small rods, } \\
\text { bunches }\end{array}$ & $\begin{array}{l}\text { Gram } \\
\text { positive, } \\
\text { short rods, } \\
\text { chains, } \\
\text { endospore } \\
\text { forming }\end{array}$ & $\begin{array}{l}\text { Gram } \\
\text { negative, } \\
\text { short } \\
\text { rods, } \\
\text { bunches }\end{array}$ & $\begin{array}{l}\text { Gram } \\
\text { negative, } \\
\text { short rods, } \\
\text { bunches }\end{array}$ & $\begin{array}{l}\text { Gram } \\
\text { negative, } \\
\text { small } \\
\text { rods, } \\
\text { bunches }\end{array}$ & $\begin{array}{l}\text { Gram positive, } \\
\text { small rods, } \\
\text { bunches }\end{array}$ \\
\hline \multicolumn{2}{|c|}{ Endospore staining } & - & - & - & + & - & - & - & - \\
\hline \multicolumn{2}{|c|}{ Motility } & + & - & - & + & + & - & - & - \\
\hline \multicolumn{2}{|l|}{ Catalase } & - & - & + & + & - & - & - & - \\
\hline \multicolumn{2}{|c|}{ Starch hydrolysis } & - & - & - & + & - & - & - & - \\
\hline \multicolumn{2}{|c|}{ Citrate } & + & - & - & - & - & - & - & + \\
\hline \multicolumn{2}{|l|}{ Urease } & - & + & + & - & - & + & - & + \\
\hline \multicolumn{2}{|l|}{ Oxidase } & + & + & + & - & - & + & + & + \\
\hline \multicolumn{2}{|l|}{ Indole } & - & - & - & - & - & - & - & - \\
\hline \multicolumn{2}{|c|}{ Methyl red } & - & - & - & - & - & - & - & - \\
\hline \multicolumn{2}{|c|}{ Voges Proskauer } & - & - & - & - & - & - & - & - \\
\hline \multirow[t]{2}{*}{$\begin{array}{l}\text { Nitrate } \\
\text { reductase }\end{array}$} & $\begin{array}{l}\text { Without } \\
\mathrm{Zn}\end{array}$ & - & - & - & + & - & - & - & - \\
\hline & With Zn & + & + & + & & + & + & + & + \\
\hline \multirow{4}{*}{$\begin{array}{l}\text { Triple } \\
\text { sugar iron } \\
\text { agar } \\
\text { Tentative } \\
\text { identificatio }\end{array}$} & Glucose & - & + & - & - & - & + & - & + \\
\hline & Lactose & + & + & + & - & + & + & - & + \\
\hline & Sucrose & + & + & + & - & + & + & - & + \\
\hline & & $\begin{array}{l}\text { Not } \\
\text { identified }\end{array}$ & $\begin{array}{l}\text { Actinobacillus } \\
\text { lignieresii }\end{array}$ & $\begin{array}{l}\text { Pasturella } \\
\text { ureae }\end{array}$ & $\begin{array}{l}\text { Not } \\
\text { identified }\end{array}$ & $\begin{array}{l}\text { Erwinia } \\
\text { herbicola }\end{array}$ & $\begin{array}{l}\text { Actinobacillus } \\
\text { lignieresii }\end{array}$ & $\begin{array}{l}\text { Kingella } \\
\text { kingae }\end{array}$ & $\begin{array}{l}\text { Actinobacillus } \\
\text { lignieresii }\end{array}$ \\
\hline \multicolumn{2}{|c|}{ Similarity index } & & 0.75512 & 0.70177 & & 0.41934 & 0.99086 & 0.74427 & 0.75467 \\
\hline
\end{tabular}

Table 4: Biochemical analyses for tentative identification of isolated organism

\begin{tabular}{|c|c|c|c|c|c|c|}
\hline Isolate & A30 & A31 & A32 & $\mathbf{A 3 3}$ & A34 & A35 \\
\hline Gram's staining & $\begin{array}{l}\text { Gram positive, } \\
\text { small rods, } \\
\text { bunches }\end{array}$ & NA & $\begin{array}{l}\text { Gram negative, } \\
\text { single, small } \\
\text { rods, bacilli }\end{array}$ & $\begin{array}{l}\text { Gram } \\
\text { positive, } \\
\text { small rods, } \\
\text { bunches }\end{array}$ & $\begin{array}{l}\text { Gram negative, } \\
\text { rods, bunches }\end{array}$ & $\begin{array}{l}\text { Gram negative, } \\
\text { short rods, } \\
\text { bunches \& } \\
\text { endospore } \\
\text { forming }\end{array}$ \\
\hline
\end{tabular}

\begin{tabular}{|c|c|c|c|c|c|c|c|}
\hline \multicolumn{2}{|c|}{ Endospore staining } & - & - & - & - & - & + \\
\hline \multicolumn{2}{|c|}{ Motility } & + & - & - & - & - & - \\
\hline \multicolumn{2}{|l|}{ Catalase } & + & + & - & -- & - & - \\
\hline \multicolumn{2}{|c|}{ Starch hydrolysis } & - & - & - & - & - & + \\
\hline \multicolumn{2}{|c|}{ Citrate } & - & - & - & - & - & - \\
\hline \multicolumn{2}{|l|}{ Urease } & - & + & + & - & - & + \\
\hline \multicolumn{2}{|l|}{ Oxidase } & + & + & + & - & + & + \\
\hline \multicolumn{2}{|l|}{ Indole } & + & - & - & - & - & - \\
\hline \multicolumn{2}{|l|}{ Methyl red } & - & - & - & - & - & - \\
\hline \multicolumn{2}{|c|}{ Voges Proskauer } & - & - & - & - & - & - \\
\hline \multirow{2}{*}{$\begin{array}{l}\text { Nitrate } \\
\text { reductase }\end{array}$} & Without Zn & - & - & - & - & - & - \\
\hline & With Zn & + & + & + & + & + & + \\
\hline \multirow{3}{*}{$\begin{array}{l}\text { Triple } \\
\text { sugar iron } \\
\text { agar }\end{array}$} & Glucose & - & - & + & - & - & + \\
\hline & Lactose & + & + & + & - & - & + \\
\hline & Sucrose & + & + & + & - & - & + \\
\hline \multicolumn{2}{|c|}{ Tentative identification } & Not identified & Agrobacterium & $\begin{array}{l}\text { Actinobacillus } \\
\text { lignieresii }\end{array}$ & Not identified & Kingella kingae & $\begin{array}{l}\text { Actinobacillus } \\
\text { lignieresii }\end{array}$ \\
\hline \multicolumn{2}{|c|}{ Similarity index } & & 0.52365 & 0.55006 & & 0.71915 & 0.50123 \\
\hline
\end{tabular}


After their confirmation as Actinobacillus, fermentative activity was evaluated in peptone water (Difco) with Andrade's indicator and each of the following carbohydrates (Difco): mannose, arabinose, xylose, sucrose, maltose, lactose, trehalose, mannitol, and salicin

Table 5: Fermentative reaction of Actinobacillus lignieresii

\begin{tabular}{lcc}
\hline Carbohydrate & $\mathbf{2 4} \mathbf{h r s}$ & $\mathbf{4 8 h r s}$ \\
\hline Glucose & + & + \\
Xylose & + & + \\
Mannitol & + & + \\
Lactose & - & - \\
Sucrose & + & + \\
Maltose & + & + \\
Trehalose & - & - \\
Salicin & - & - \\
Arabinose & + & + \\
\hline
\end{tabular}

\section{Molecular Analysis}

\section{Genomic DNA isolation}

Genomic DNA was extracted from overnight grown culture in Luria broth. $1.5 \mathrm{ml}$ of culture was taken in fresh autoclaved eppendorf tube and centrifuged at $10000 \mathrm{rpm}$ for $5 \mathrm{~min}$. The supernatant was discarded and pellet was resuspended in $500 \mu \mathrm{l}$ of TAE buffer and vortexed. $10 \mu \mathrm{l}$ of lysozyme $(20 \mu \mathrm{g} / \mathrm{ml})$ and $40 \mu \mathrm{l}$ of $10 \%$ SDS was added and incubated at $4^{0} \mathrm{C}$ for $15 \mathrm{~min}$. Equal volume of Tris saturated phenol was added and tubes were inverted gently 2-3 times. Centrifugation was done at $12000 \mathrm{rpm}$ for $15 \mathrm{~min}$. The upper aqueous layer, containing DNA was transferred to fresh eppendorf. Equal volume of chloroform: isoamyl alcohol (24:1) was added \& mixed by inverting the tubes 4-5 times gently. Tubes were centrifuged at $10000 \mathrm{rpm}$ for $10 \mathrm{~min}$. Upper aqueous layer was taken $\&$ transferred to fresh tube and chilled absolute alcohol was added. Tubes were inverted gently 2-3 times \& centrifuged at $10000 \mathrm{rpm}$ for $10 \mathrm{~min}$. Supernatant was discarded and pellet was washed with $70 \%$ alcohol at $10000 \mathrm{rpm}$ for $10 \mathrm{~min}$. After centrifugation alcohol was removed completely and pellet was allowed to dry for

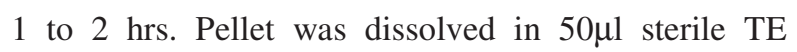
buffer. The isolated DNA was checked by running them on $0.8 \%$ agarose gel with $\lambda$ DNA marker.

\section{Amplification of 16S rRNA}

Amplification of $16 \mathrm{~S}$ rRNA region was done using PCR with degenerate primers, 27F (5'AGAGTTTGATCMTGGCTCAG-3') and 519R (5-' GWATTACCGCGGCKGCTG-3') specific to conserved regions. The PCR reaction was done using a BioRad thermal cycler. The volume of reaction mixture was $25 \mu \mathrm{l}$, containing $8 \mu \mathrm{l}$ of genomic DNA, $2.5 \mu \mathrm{l}$ of $10 \mathrm{X}$ PCR buffer (10 mM Tris- $\mathrm{HCl}$; $\mathrm{pH}$ : 9.0, $50 \mathrm{mM} \mathrm{KCl}, 1.5 \mathrm{mM} \mathrm{MgCl}_{2}$ ), $1 \mu \mathrm{l}$ of Taq DNA polymerase, $1 \mu \mathrm{l}$ of $10 \mathrm{mM}$ dNTPs mix and $2 \mu \mathrm{l}$ each of forward and reverse primers (Bangalore Genei, India). The PCR conditions were standardized as follows: initial denaturation at $94^{\circ} \mathrm{C}$ for $3 \mathrm{~min}, 30$ cycles of denaturation at $94^{\circ} \mathrm{C}$ for $40 \mathrm{~s}$, annealing at $50^{\circ} \mathrm{C}$ for $45 \mathrm{~s}$ and primer extension at $72^{\circ} \mathrm{C}$ for $1 \mathrm{~min}$ and final extension at $72^{\circ} \mathrm{C}$ for $7 \mathrm{~min}$. The amplified products were analyzed on a $0.8 \%$ agarose gel with ethidium bromide.

\section{Results}

\section{Morphological and biochemical analysis}

A total of 30 different morphological forms were isolated from the cattle waste compost collected from Jaipur, India. The ability of these isolates to grow at different temperatures, $\mathrm{pH}$ and salt concentrations was studied to find their optimal growth condition. The isolates A3, A4, A10, A15, A18, A20, A21, A25, A26, A29, A30, A31, A32 and A35 showed maximum growth at $55^{\circ} \mathrm{C}$ and the isolates $\mathrm{A} 1, \mathrm{~A} 5, \mathrm{~A} 6, \mathrm{~A} 7, \mathrm{~A} 8$, A9, A11, A13, A14, A17, A19, A23, A33 and A34 at $60^{\circ} \mathrm{C}$ (Fig.1).

The optimum temperature of growth is $70^{\circ} \mathrm{C}$ for the isolates A20, A30 and A32. None of the isolates showed growth at $35^{\circ} \mathrm{C}$ but all strains had growth at $60^{\circ} \mathrm{C}$ revealing their thermophilic character and can be classified as moderate thermophiles (Gomes et al., 2004). These bacteria are highly active at $60^{\circ} \mathrm{C}$ and at temperatures above $60^{\circ} \mathrm{C}$ the degradation process is performed by these microorganisms (Fogarty et al., 1991; Finstein et al., 1975; Nakasaki et al., 1985a; Strom, 1985b; Sharp et al., 1992). Microbial diversity is a prerequisite for a satisfactory composting process. High temperature more than $60^{\circ} \mathrm{C}$ are considered to reduce dramatically the functional diversity. It is generally assumed that to obtain an efficient and rapid decomposition, temperature should not be allowed to exceed 55 to $60^{\circ} \mathrm{C}$ (Mckinley et al., 1984; Turner et al., 2007). Of the 30 isolates, 14 having good growth at 55 and $60^{\circ} \mathrm{C}$ were further used to study the effect of $\mathrm{pH}$ and salt concentration. All the isolates showed optimal 
Saggu GS and Shrivastava D (2013). Int J Appl Sci Biotechnol, Vol. 1(2): 33-41

growth at pH 7 whereas isolates A15 and A20 showed optimal growth at pH 9 (Fig. 2).

In the composting process, initially $\mathrm{pH}$ goes down because of acid production and then increases by ammonia production. So the isolates are adapted to neutral pH. Four isolates (A10, A25, A29 and A30) showed optimum growth at $1 \%$ salt concentration. All isolates were able to grow at $2 \% \mathrm{NaCl}$ concentration compared to higher salt concentration. Isolate A9 was unable to tolerate even $1 \%$ salt concentration. Isolate A21 showed growth up to $7 \%$ salt concentration (Fig: 3).

These isolates were identified on the basis of morphological, physiological \& biochemical characteristics using software for probabilistic identification of bacteria (http://www.som.soton.ac.uk/staff/tnb/pib.htm).

Colonies were non haemolytic, small, raised, and greyish white. Majority of organisms stained as pleomorphic gram-negative rods. Out of 30 isolates $66.6 \%$ were gram negative and the rest were found to be gram positive. Spore formation was observed in 5 gram positive isolates (A5, A7, A17, A19 and A23) and 5 gram negative isolates (A1, A8, A9, A14, A35) (Table: 1-4). Heat resistant spores were found among several species, mainly those belonging to the genera
Bacillus and Clostridium (Ferna'ndeza et al., 2001; Hyun et al., 1983). Less attention has been paid to thermophilic heat resistant spores than mesophilic spores which are used in food industry. Spores from thermophilic bacteria are more heat resistant than spores from mesophilic species (Warth, 1978). Protease and cellulases activity is not there in any of the isolates. These 30 isolates were identified to belong to 8 genera namely Actinobacillus, Pasteurella, Micrococcus, Brahnamella, Erwinia, Kingella, Bordetella and Agrobacterium. Of these 18 isolates were identified up to species level using the software (http://www.som.soton.ac.uk/staff/tnb/pib.htm). 8 isolates were identified as A. lignieresii, 3 isolates as Pasteurella haemolytica, 2 isolates as Pasteurella ureae, 2 isolates as Erwinia herbicola, 2 isolates as Kingella kingae and one isolate as Streptococcus cremoris. Isolates which were regarded as a possible Actinobacillus after initial biochemical tests were further evaluated for fermentative activity. The isolates were inoculated into peptone water with Andrade's indicator and each of the following carbohydrates: mannose, arabinose, xylose, sucrose, maltose, lactose, trehalose, mannitol, and salicin. We used the differential identification scheme because Actinobacillus identification is very confusing.

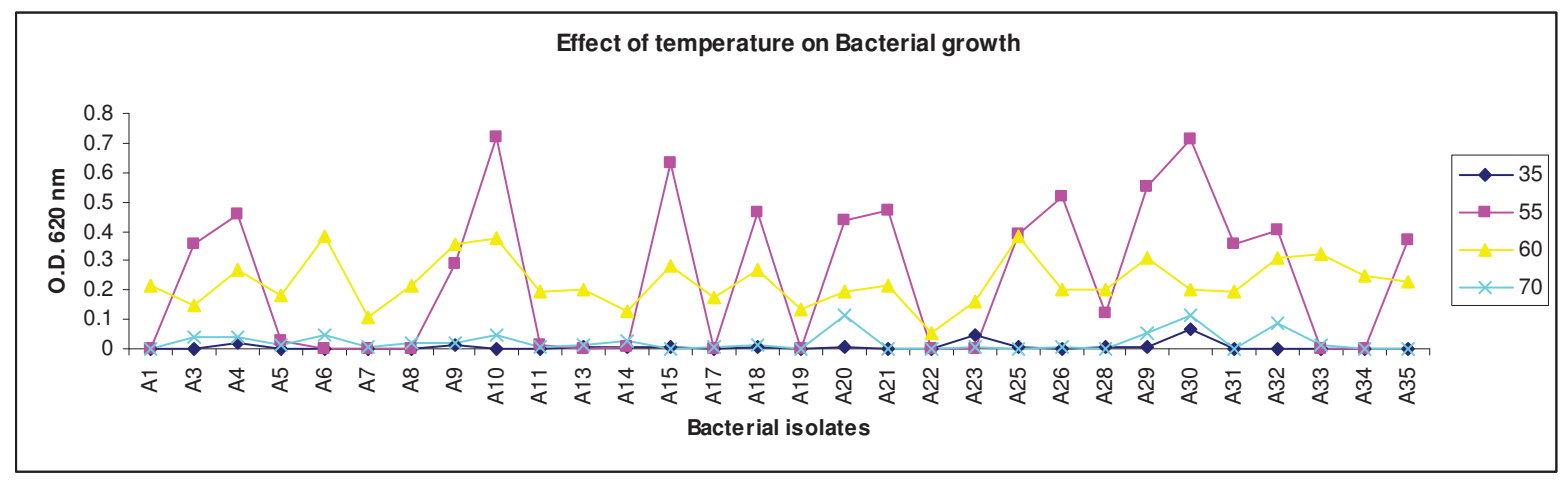

Fig. 1: Effect of temperature on bacterial isolates.

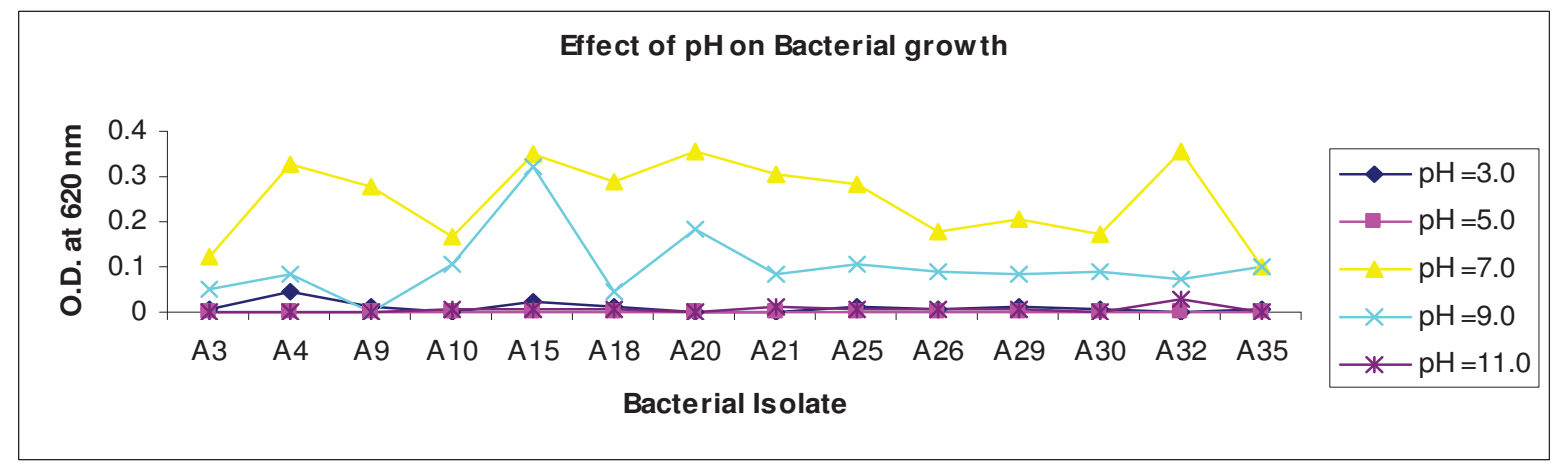

Fig. 2: Effect of $\mathrm{pH}$ on bacterial isolates. 


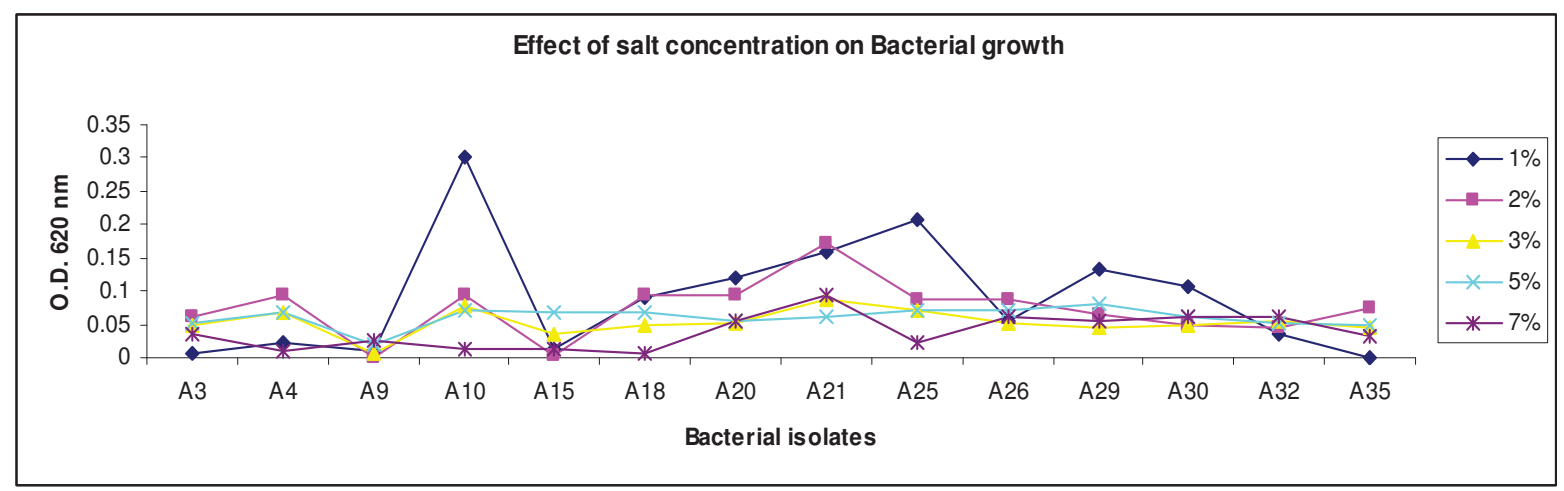

Fig. 3: Effect of salt concentration on bacterial isolates.

\section{$16 S$ rRNA region amplification and sequencing}

The DNA was isolated from these eight isolates of Actinobacillus which were unable to grow below $55^{\circ} \mathrm{C}$ indicating their nonpathogenic nature. The $16 \mathrm{~S}$ rRNA region of $991 \mathrm{bp}$ amplified from these isolates by using $512 \mathrm{R}$ as a reverse primer and $27 \mathrm{~F}$ as a forward primer (Fig 4).

Sequencing has been done and submitted, for the amplified region to Genebank of National Central for Biotechnology. Nucleotide sequence data reported in this paper are available in the Genebank, EMBL and DDBJ databases under the accession number: JQ783352

\section{Discussion}

The study was designed to study thermophiles but after experimental analysis we found that Actinobacillus which generally survive in moderate temperature can sustain higher temperature also. It has been found that Actinobacillus was the most dominant bacteria in cattle compost. A. lignieresii is responsible for actinobacillosis, an infectious, chronic, generally nonfatal disease (Rebhun et al., 1988). In cattle, it typically infects the tongue and hence is also known as "the Wooden Tongue" (Misra et al., 1981; Mohanty et al., 1970). The involvement of other organs is considered to be atypical (Aslani et al., 1995; Holzhauer et al., 2002; Rebhun et al., 1988; Mallick et al.,1984; Misra et al.,1981). The etiologic agent is found normally in the oropharynx and rumen of cattle and sheep (Smith, 1990; Songer et al., 2005). The organism is an opportunistic pathogen and it causes chronic pyogranulomatous lesions of the soft tissues of head and neck regions in cows, buffalo, sheep, goats, and horses (Baum et al., 1984; Fubini et al., 1983; Hirsh et al., 2004; Kumar et al., 1998; Songer et al., 2005). Clinically, the lesions appear as nodules, multiple abscesses, ulcers or draining fistulae. A. lignieresii is causative agent for spontaneous actinobacillosis in the buffalo (Bubalus bubalis) which reported as non-fatal (Ahmad et al., 1986; Akhtar et al., 1964). In previous studies A. lignieresii is already described as causative agent of a granulomatous disease of cattle and it was also responsible for the prevalent form of the similar. Report shows that Actinobacillus can also cause infection in human which escort to death (Beaver et al., 1933) but no recent reports are available for that.

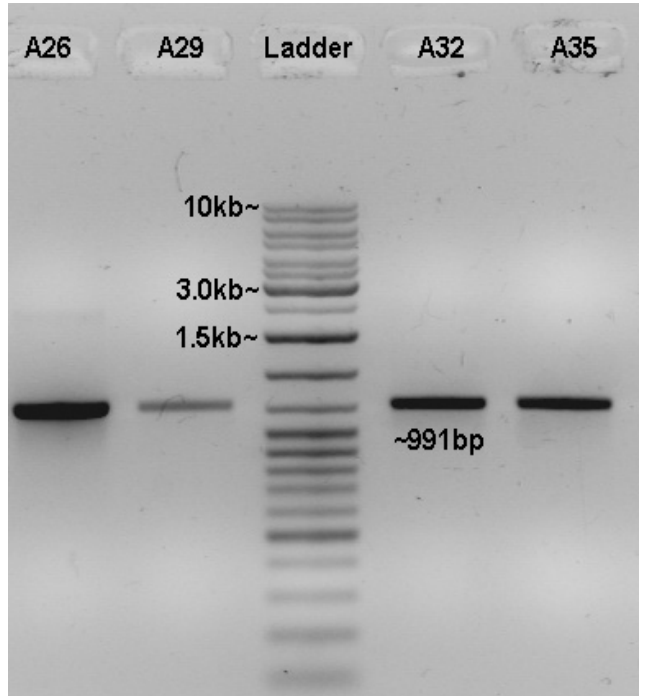

Fig. 4: A. lignieresii DNA amplified by $519 \mathrm{R}$ and $27 \mathrm{~F}$ primer

Isolation of Actinobacillus has been already done from laboratory rodents, (Lentsch et al., 1980; Simpson et al., 1980) but characterization of these organisms is still mystery; host range for the organism is still incomplete. Optimum growth condition for Actinobacillus is $37^{\circ} \mathrm{C}$ temperature and acidic $\mathrm{pH}$ but in this study we found that it shows adaptation for $60^{\circ} \mathrm{C}$ temperature also. Justification can be given as that during composting process temperature rises 60 to $70^{\circ} \mathrm{C}$ and Actinobacillus make itself to survive in those conditions. Actinobacillus also shows degradation ability in these high temperature conditions. Amino acid composition of surface proteins in mesophylic and thermophilc bacteria is different, which responsible for the survival in harsh condition, which shows that different set of proteins, are expressed in these conditions. Protein expression and different metabolic activity of Actinobacillus in these conditions is yet to 
be studied. These days' researchers are more interested in proteins which are expressed in those organisms which helps them to sustain that kind of environment. Till date there is no report for protein expression studies on Actinobacillus spp. According to our knowledge this is the first report of Actinobacillus from India in which one pathogenic organism got adapted to thermophilic nature and lost its pathogenecity. This study also generates several questions in our mind like; how a mesophilic pathogenic organism converted to non pathogenic thermophilic organism. Expression of different set of gene is required to sustain that environment and what were the factors which functions as an initiator. Another big question is how organism lost its pathogenecity after acquiring that adaptation.

\section{Acknowledgement}

Authors equally contributed for this work and there is no conflict of interest. Jaipur National University, Jaipur, Rajasthan provided the basic lab requirement and funds for this work. We acknowledge our professors for their motivation and direction for this work.

\section{References}

Ahmad A, Majeed MA and Jabbar MA (1986) Blood picture of naturally occurring actinobacillosis in male buffalo calves. Pakistan Veterinary J. 6: 80-83.

Akhtar AS and Cheema RA (1964) Actinobacillosis in buffaloes. Abstr G-24, Proceedings of 16th Pakistan Science Conference on Medicine and Veterinary Science.

Aslani MR, Khodakaram A and Rezakhani A (1995) An atypical case of actinobacillosis in a cow. J Vet Med. 42: 485-488.

Baum KH, Shin SJ, Rebhun WC and Patten VH (1984) Isolation of Actinobacillus lignieresii from enlarged tongue of a horse. J Am Vet Med Assoc. 185: 792-793.

Beaver DC and Thompson L (1933) Actinobacillosis of man* Report of a fatal case, From the Section of Pathologic Anatomy and the Section on Clinical Pathology, The Mayo Clinic, Rockester, Minn.

Brock TD (1967) Life at high temperatures. Science 158: 1012-1019.

Brock TD and Darland GK (1970) Limits of microbial existence: temperature and $\mathrm{pH}$. Science 169: 13161318.

Ferna'ndeza A, Ociob MJ, Ferna'ndezb PS and Mart'ineza A (2001) Effect of heat activation and inactivation conditions on germination and thermal resistance parameters of Bacillus cereus spores. International Journal of Food Microbiology. 63: 257-264.

Finstein M S and Morris M L (1975) Microbiology of municipal solid waste composting. Adv. Appl. Microbiol.19: 113-151.
Fogarty AM and Tuovinen OH (1991) Microbiological degradation of pesticides in yard waste composting. Microbiol. Rev. 55: 225.

Fubini SL and Campbell SG (1983) External lumps on sheep and goats. Veterinary Clinics of North America. Large Animal Practice 5: 457-476.

Gomes J and Steiner W (2004) The Biocatlytic Potential of Extremophiles and Extremozymes. Food Technol. Biotechnol. 42: 223-235.

Hirsh DS, Maclachlan NJ and Walker RL 2004. Veterinary Microbiology. (2nd eds) Blackwell Publishing Professional, Ames, Iowa, 91-94.

Holzhauer M and Roumen TP (2002) Atypical actinobacillosis in a dairy replacement herd. Vet Rec. 151: 276.

Hyun HH, Zeikus JG, Longin R, Millet J and Ryter A (1983) Ultra structure and Extreme Heat Resistance of Spores from Thermophilic Clostridium Species. J. bacteriolo. 156: $1332-1337$.

Ibrahim ASS and Diwany A (2007) Isolation and Identification of New Cellulases Producing Thermophilic Bacteria from an Egyptian Hot Spring and Some Properties of the Crude Enzyme. Aust. J. Basic Appl. Sci. 1: 473-478.

Kumar GS and Parihar NS (1998) Respiratory ailments in Indian buffaloes. Buffalo J. 14: 109-114.

Ladenstein R and Antranikian G (1998) Proteins from hyperthermophiles: Stability and enzymatic catalysis close to the boiling point of water. Advances in Biochemical Engineering and Biotechnology 61: 37-85.

Lentsch RH and Wagner JE (1980) Isolation of Actinobacillus lignieresii and Actinobacillus equuli from Laboratory Rodents. J. Clin. Microbio. 351-354.

Mallick KP, Somvansi R and Moga IV (1984) Actinobacillosis in the testicle of a buffalo. Indian $J$. Anim. health. 23: 95-96.

Mckinley VL and Vestal JR (1984) Biokinetic analyses of adaptation and succession: microbial activity in composting municipal sewage sludge. Appl. Environ. Microbiol. 47: 933-941.

Misra SS and Angelo SJ (1981) Treatment of lingual actinobacillosis in a buffalo. Indian Vet J. 58: 413- 414.

Mohanty GC and Singh CM (1970) Actinobacillotic nephritis in a male buffalo: a case report. Indian Vet J. 47: 938941.

Nakasaki K, Shoda M and Kubota H (1985a) Effect of temperature on composting of sewage sludge. Appl. Environ. Microbiol. 50: 1526-1530.

Nakasaki K, Sasaki M, Shoda M and Kubota H (1985b) Change in microbial numbers during thermophilic composting sewage sludge with reference to $\mathrm{CO}_{2}$ evolution rate. Appl. Environ. Microbiol. 49: 37-41.

Rebhun WC, King JM and Hillman RB (1988) An atypical actinobacillosis granulomas in cattle. Cornell Vet. 78: 125-130.

Schulze KL (1962) Continuous thermophilic composting. Appl. Microbiol. 10: 108-122.

Sharp RJ, Riley PW and White D (1992) Heterotrophic thermophilic Bacilli. In: Thermophilic Bacteria, In 
Saggu GS and Shrivastava D (2013). Int J Appl Sci Biotechnol, Vol. 1(2): 33-41

Kristjansson, J.K. (eds), CRC Press Inc., Boca Raton U.S.A.19-50.

Simpson W and Simmons DJC (1980) Two Actinobacillus species isolated from laboratory rodents. Laboratory Animals 14: 15-16.

Smith BP (1990) Large Animal Internal Medicine. The C.V. Mosby Co. Philadelphia, 719-721.

Satyanarayana T, Raghukumar C and Shivaji S (2005) Extremophilic microbes: Diversity and perspectives. Curr. Sci. 89: 78-90.

Songer JG and Post KW (2005) Veterinary Microbiology Bacterial and Fungal Agents of Animal Disease. ELSEVIER SAUNDERS, St. Louis, Missouri, 174-180.

Strom PF (1985a) Identification of thermophilic bacteria in solid waste composting. Applied Environ. Microbiol 50: 906-913.
Strom PF (1985b) Effect of temperature on bacterial species diversity in thermophilic solid-waste composting. Appl. Environ. Microbiol. 50: 899-905.

Suler DJ and Finstein MS (1977) Effect of temperature, aeration, and moisture on $\mathrm{CO}_{2}$ formation in benchscale, continuously thermophilic composting of solid waste. Appl. Environ. Microbiol. 33: 345-350.

Turner P, Mamo G and Karlsson EN (2007) Potential and utilization of thermophiles and thermostable enzymes in biorefining. Microbial Cell Factories 6: 9.

Warth AD (1978) Relationship between the heat resistance of spores and the optimum and maximum growth temperature of Bacillus species. J. Bacteriol. 134: 699. 